\title{
WHEN IT'S RIGHT IN FRONT OF YOU : ASSISTING HEALTH CARE WORKERS TO MANAGE THE EFFECTS OF VIOLENCE IN RURAL AND REMOTE AUSTRALIA
}

The effect of violence on the physical and mental health of individuals, families and communities can be severe and far-reaching. There are many different kinds of violence. It may be person-to-person or self-inflicted. It may be physical, sexual or verbal abuse, harassment, bullying, or discrimination. Violence does not 'belong' to any one community, culture, or group of people in Australia. It affects people of all ages and all backgrounds.

Resources for coping with the effects of violence are limited, particularly in rural and remote Australia. The National Health and Medical Research Council (NHMRC) has developed a manual, When it's right in front of you: Assisting health care workers to manage the effects of violence in rural and remote Australia, for health care workers, managers, and employer organisations in rural and remote Australia. The manual has been designed to assist in preparing for and respond to violence in ways that will minimise its impact.

The manual addresses the two main categories of violence that health care workers and managers may encounter. These are: violence suffered by clients, and occupational violence. The manual is a tool to assist and guide in the management of episodes of violence. It provides practical guidance, and identifies useful Web sites and references that provide more specific information. It does not provide legal advice. It encourages partnerships and capacity building at a local level, recognising the variety of employers, settings, and circumstances in different rural and remote areas.

The manual was prepared by a working party of the NHMRC Health Advisory Committee and is based on a review of literature on the epidemiology of violence and resources relevant to the management of violence by health care workers in rural and remote Australia. The manual was the subject of extensive consultation including conference and seminar presentations, a request for public submissions, and a workshop involving practitioners with experience in rural health care.

The manual is available on the NHMRC website at www.nhmrc.gov.au and from the NHMRC Health Advisory Section on (02) 6289 9814.

\section{WORLD REPORT ON VIOLENCE AND HEALTH}

The World Report on Violence and Health was published by the World Health Organization in October 2002. The Report aims to:

- increase attention to violence as a major social and health problem;

- draw attention to the contribution that the public health approach can make to understanding and responding to violence;

- increase the commitment by the public health community to addressing violence.

The Report presents state of the art reviews of child abuse, sexual violence, youth violence, intimate partner violence, elder abuse, collective violence, and suicide. It presents a model to understand violence that focuses attention on individual, relationship, community, and societal level risk factors and intervention possibilities. It highlights the common risk factors for different types of violence, and promotes greater communication between those specialising in single types of violence.

The key recommendations of the Report are to:

- create, implement and monitor a national action plan for violence prevention;

- enhance capacity for collecting data on violence;

- define priorities for, and support research on the causes, consequences, costs and prevention of violence;

- promote primary prevention responses;

- strengthen responses for victims of violence;

- integrate violence prevention into social and educational policies, and thereby promote gender and social equality;

- increase collaboration and exchange of information on violence prevention;

- promote and monitor adherence to international treaties, laws and other mechanisms to protect human rights;

- seek practical, internationally-agreed responses to the global drugs trade and the global arms trade.

A recent issue of the Australian and New Zealand Journal of Public Health (Volume 26, Number 5) devotes attention to the Report and presents Australian perspectives on violence in the indigenous community, violence against women, child abuse, elder abuse, and the role of alcohol. In addition, a conference Violence and Health : Australian responses, to be held at the University of New South Wales on 18 November, will debate the report, and present data from leading Australian researchers and policy makers, on violence. The conference will also consider how to best move the field forward.

The Report can be accessed online at www.who.int/violence_injury_prevention. 